\title{
Efficient Data Access in Vehicular Ad Hoc Networks
}

\author{
Anjali \\ Student, Computer Science \\ Lovely Professional University \\ Phagwara, India
}

\author{
Mohinder Kumar \\ Assistant Professor, Computer Science \\ Lovely Professional University \\ Phagwara, India
}

\begin{abstract}
VANET is a highly movable wireless ad hoc network that is meant to support road safety, monitoring the traffic etc. In VANET environment, each vehicle can go for access to data from RSU or can communicate to any other vehicle. In this scenario, service scheduling becomes an important challenge to provide equal distribution of data access. "Data Scheduling" measured a very important issue in VANET to successful delivery of data item to the vehicle in proper and accurate way. Communication among vehicles are becoming a promising technology for security, management of traffic, monitoring and controlling of pollution, and numerous other road safety and traffic applications. Due to this a lot of data is generated that must be shared between communication parties efficiently. A major load is caused on the network infrastructure because of all this generated data, and the main aim of the network infrastructure is to provide constant services to the users. Thus, in order to manage the load on the network in such situations, a new scheme is proposed which suggests to cache frequently accessed contents at particular locations such as vehicles and RSUs so that data can be accessed from either local cache or RSU cache without the need to flood requests for the required data in the whole network thus reducing the delay and ultimately increasing the throughput.
\end{abstract}

\section{Keywords}

Vehicular Ad Hoc Network (VANET), Peer to peer (P2P), Vehicle to Infrastructure (V2I), Vehicle to Vehicle (V2V) Fast Interacting $\mathrm{Ad} \mathrm{Hoc}$ on Demand Distance Vector (FIAODV).

\section{INTRODUCTION}

Vehicular Ad-hoc Networks (VANETs) are going up as is the favourite network plan for intelligent transportation systems. VANETs are based on short range wireless exchange and share of information between vehicles. By installing on-board transceivers in the vehicles, recently growing up VANET facilitates wireless communication among vehicles and roadside units, for internet access using the special DSRC (Dedicated Shortest-Range Communications) radio spectrum.

A VANET is applicable on vehicles moving on road. It is envisaged for usage in multiple areas like safety of road, convenience in moving of vehicles on road, and business purpose. Out of them, data dissemination is a significant kind. Exhaustive investigation is carried out to efficiently disseminate data to many beneficiaries in the network. It is observed that main object of data dissemination applications is to approach to required number of recipients economically.

DSRC (Dedicated Short-Range Communication) provides the ability to efficiently carry out inter-vehicular sharing and exchange of messages etc that is known as technology of Vehicle Safety Communication. With short-range communication having $5.9 \mathrm{GHz}$ frequency, vehicles will attain ability to communicate with each other and also with their surroundings. Numerous provisions will be provided for networks of vehicles which help to increase the protection of the infrastructure of transportation. For example, the system will achieve ability to synchronize traffic lights in order to make the flow of traffic smooth.

Now, due to the rapid advancements in the high-speed internet, it is possible for the users to access any kind of services while sitting in their vehicles, such as users can control their household equipments remotely when they are away. This is all due to the development of a new rising technology i.e. vehicular ad hoc networks in which vehicles are equipped with on board units which perform a variety of functions such as collecting the data and forwarding it to the other vehicles.

There are two types of communications in VANETs i.e. vehicle-to-vehicle (V2V) and vehicle-to-infrastructure (V2I) communications. Vehicles can access a variety of resources from the nearby RSUs in vehicle to infrastructure (V2I) communications these RSUs act as access routers which establishes connection of the vehicles with the network infrastructure. RSUs are installed along the roads and act as intermediary between the network infrastructure and vehicle for the purpose of data sharing among the vehicles in both the directions. On the other hand there is direct communication between the vehicles in case of vehicle to vehicle (V2V) communication without the involvement of RSUs. While in motion, vehicles share important information such as road traffic, weather conditions, or security alarms with each other either in a cooperative manner or with the help of installed RSUs.

\section{TRAFFIC MODEL AND PROBLEM FORMULATION}

\subsection{Traffic Model}

2-D traffic model is considered in our scheme. In this scheme, clients can go bidirectional and frequently used information can either be located at RSU, at a gateway, or in vehicles. A gateway is used in the internet to offer a variety of services to the moving vehicles for example users can either send or receive the data while in motion.

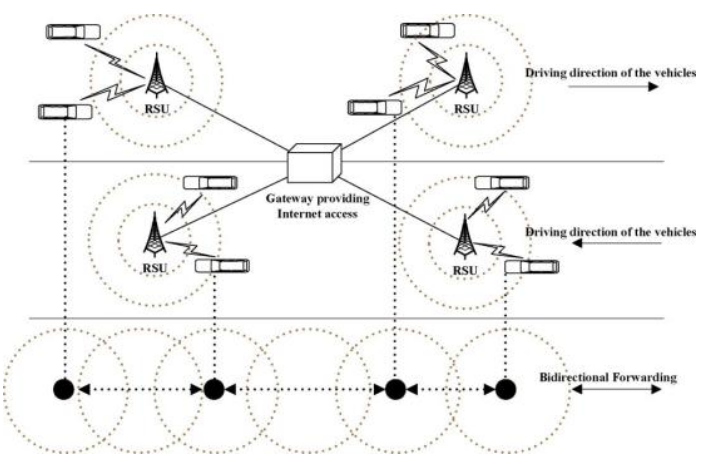

Fig. 1: Considered Traffic Model. 
RSUs are fixed in multiple locations in the case of vehicle to infrastructure communication. The data is sent or received to the server constantly or after some fixed time intervals. In case of vehicle to vehicle communication, every vehicle sends and receives data from one another cooperatively. In the proposed scenario first kind of communication is considered in which there is communication of vehicles among themselves as well as with RSU for information sharing without burdening the server due to which throughput of the system is improved to great extent.

\subsection{Problem Formulation}

Let $V=\{V 1, V 2, \ldots, V n\}$ denote the vehicles in the network. $D=\{D 1, D 2, \ldots, D n\}$ and $Q=\{Q 1, Q 2, \ldots, Q n\}$ are respectively the identifiers for the data (IDs) and requests made for particular information which can be cached during the period of communication. Every request for a particular information has the following metrics $\left\{V_{i}, D_{i}, \theta, \rho, \tau_{\text {interval }}\right.$, where $\theta$ and $\rho$ respectively are the rate of use and probability of accessibility of the information in a time interval $\tau_{\text {interval }}$. The request is sent to RSU under which a vehicle $V_{i}$ falls. When RSU receives the request for a particular data, it looks into its local cache for the requested data. If the requested data is there in its local cache, then the RSU replies. Else, the request enters into a waiting state. If after some time the requested data is available, the request is responded to the vehicle which sent it, by the time RSU will send same query to other RSU to find and locate data item $\mathrm{D}_{\mathrm{i}}$. Every request's progress is also observed by the start time $\tau_{\text {start }}$ and update time $\tau_{\text {update }}$. The time interval for handling the request is considered as the difference between $\tau_{\text {start }}$ and $\tau_{\text {update }}$.

\subsection{Flow Chart of Proposed Scheme}

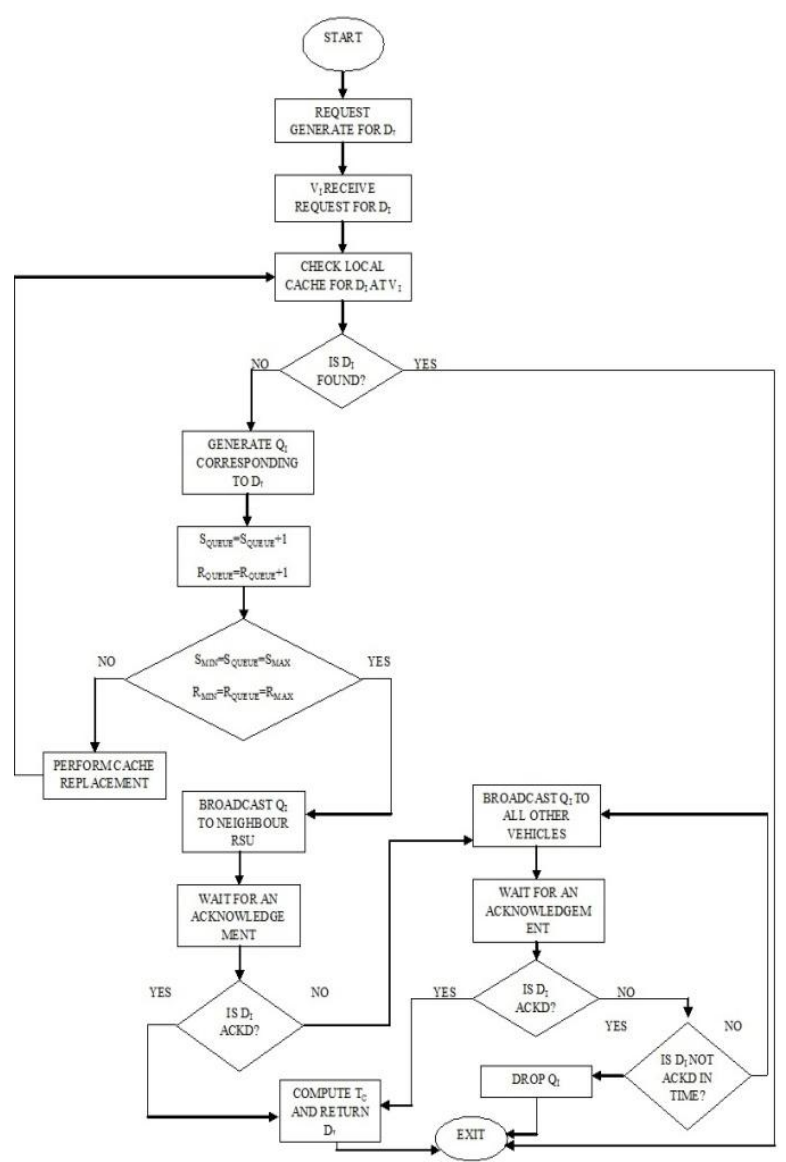

\section{SYSTEM MODEL}

\subsection{Overview}

$\mathrm{P} 2 \mathrm{PCC}$ in V2I communication is adopted, i.e. cache will now also be maintained at RSU and vehicle $\mathrm{V}_{\mathrm{i}}$ demanding for data $D_{i}$ will first search for data in its local cache, in case it does not have that data, it will ask for $\mathrm{RSU}_{\mathrm{i}}$ under whose range vehicle $\mathrm{V}_{\mathrm{i}}$ comes to provide with the data, if the data will be available in the cache of $\mathrm{RSU}_{\mathrm{i}}$ it will send it back otherwise, will perform RSU to RSU communication to ask for the data $\mathrm{D}_{\mathrm{i}}$ from other RSUs, as the data will be received by query generating RSU i.e. $\mathrm{RSU}_{\mathrm{i}}$ it will serve the demand of vehicle $\mathrm{V}_{\mathrm{i}}$ demanding for it. In this manner, load on the network infrastructure will be reduced as V2I communication will help serving more requests per unit time than $\mathrm{V} 2 \mathrm{~V}$ because RSUs will be having more processing speed than a vehicle, largesized cache than a vehicle and much more battery power, thus if more number of data requests will be served per unit time, dissemination task will be done faster leading to lower dissemination delay and thus increasing the throughput w.r.t. Packet Delivery Ratio.

\subsection{Algorithm}

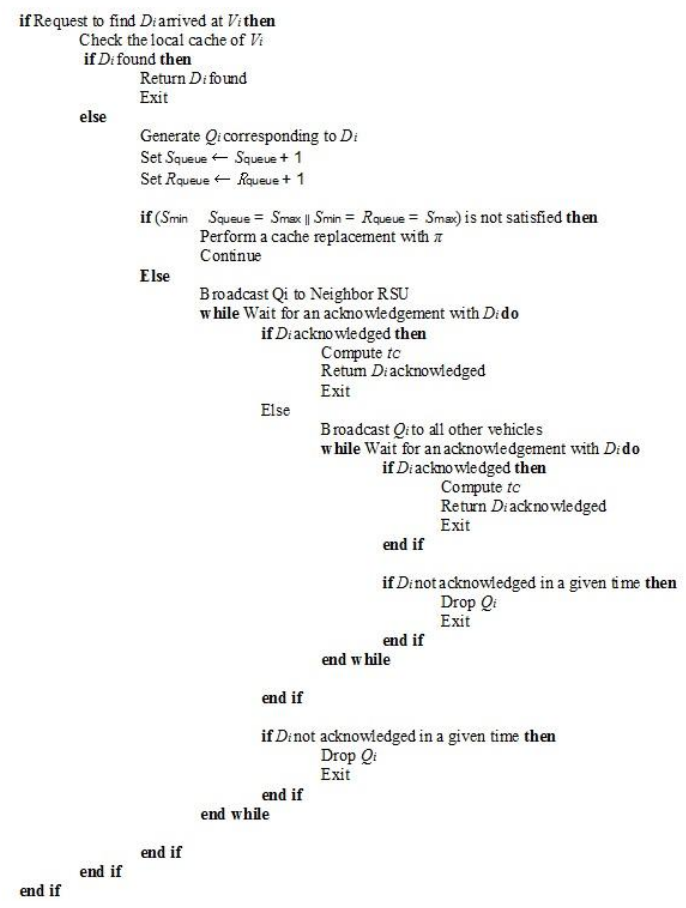

Firstly, a query for the requested data id is generated by the user. A queue is maintained, in which the upper and lower values are set for every vehicle, in order to keep track of all the requests created either for same data ID or different data ID. Depending upon the length of queue i.e. if the length is between the upper and lower fixed value, the queue is accepted and the data item for which the query is generated is searched and the time to start the query is set. If the vehicle could not find the searched data item in its local cache then it disseminates the query generated for particular information to all the vehicles and waits for a particular time of the acknowledgement from other vehicles.

Following are the steps of the algorithm: A request is transmitted to the all RSUs along with the ID of the vehicle and an acknowledgement is waited. In case, if there is no reply received then the request is discarded, otherwise every intermediary RSU looks into the cache which every RSU maintains locally, for searching the requested data in it. If the 
data is found then the RSU replies back, or transmit the results from one RSU to another in a cooperative manner. In a particular region, all the RSUs are searched for the data. Now, in the second phase after receiving the acknowledgement, space is made available for new data. If there is not enough space to accommodate the new data, then the nearby RSU is requested. If the nearby RSU responds to the request sent then the message is transmitted to the requesting vehicle. Else the vehicle enters into the update state and the value of time is incrementing. In this way the queries are replaced in the cache of vehicle. If the size of the queue is larger than the threshold value, then it enters into the waiting state and few data items in the cache are replaced with the ID of vehicle, rate of use and ID of data. The replacement of cache needs the ID of vehicle, rate of data use, and ID of the data. For every data, probability is calculated using (I), and that data item with a least value of the probability is replaced as computed in (I).

$$
\pi=\frac{1}{t_{w} D_{i} \theta \tau_{\text {interval }}}
$$

\section{RESULTS AND DISCUSSION}

\subsection{Simulation Environment}

The tool used for simulating and evaluating the proposed scheme is Network Simulator 2. Network Simulator 2 is an open source and very famous simulator. NS2 is an objectoriented, event driven network simulator. It uses $\mathrm{C}++$ and $\mathrm{Tcl}$. These two programming languages are used for a reason. The interior features of these two programming languages are the main reason of why they are used. C++ is capable of implementing the proposed scheme, but showing the results visually and graphically is not easy in $\mathrm{C}++$. In the field of research in networks, organizing a complete test environment consisting of many connected computers, data connections and routers will become very expensive to verify the results of a protocol or algorithm. In these situations, network simulators can help completing this task that too in cost and time efficient manner. Network simulator also allows the designers of networks to test the existing or changed protocols in a managed way. In general, real world networks are tried to be modeled by the network simulators. The basic idea is that by creating a model of the system, its characteristics can be altered and the consequent results can be examined. The metrics of simulation are provided in Table I.

Table I. Used Parameters and Values

\begin{tabular}{|l|l|}
\hline PARAMETER & VALUE \\
\hline $\begin{array}{l}\text { NUMBER OF VEHICLES } \\
\text { (NODES) }\end{array}$ & 500 \\
\hline $\begin{array}{l}\text { AVERAGE VELOCIIY OF } \\
\text { VEHICLES }\end{array}$ & $20-40 \mathrm{miles} / \mathrm{h}$ \\
\hline PERIOD OF TRAFFIC LIGHTS & $60 \mathrm{sec}$ \\
\hline $\begin{array}{l}\text { WIRELESS COMMUNICATION } \\
\text { RANGE }\end{array}$ & $300 \mathrm{~m}$ \\
\hline QUERY INTERVAL & $10 \mathrm{sec}$ \\
\hline SIZE OF NODE CACHE & $4 \mathrm{MB}$ \\
\hline SIZE OF RSU CACHE & $8 \mathrm{MB}$ \\
\hline
\end{tabular}

\subsection{AODV Vs FIAODV}

We have evaluated the proposed scheme and performed the comparison with the existing AODV protocol in terms of Packet Delivery Ratio, throughput, delay. Figure 2 shows the variation of the packet loss in both AODV and FIAODV, Figure 3 shows the variation in the throughput of both the schemes and Figure 4 shows the variation in the delay of both the schemes. As shown, the proposed scheme, FIAODV, has better performance in terms of all the above mentioned parameters as compared to the AODV.

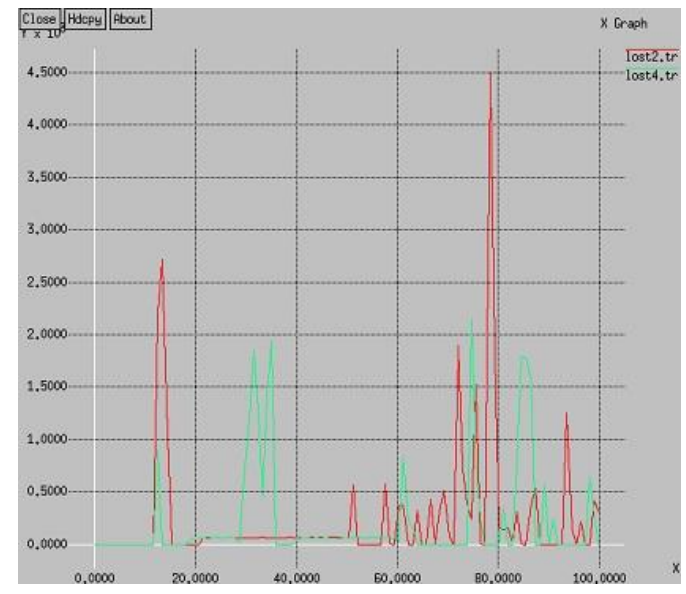

Fig. 2: Packet Loss

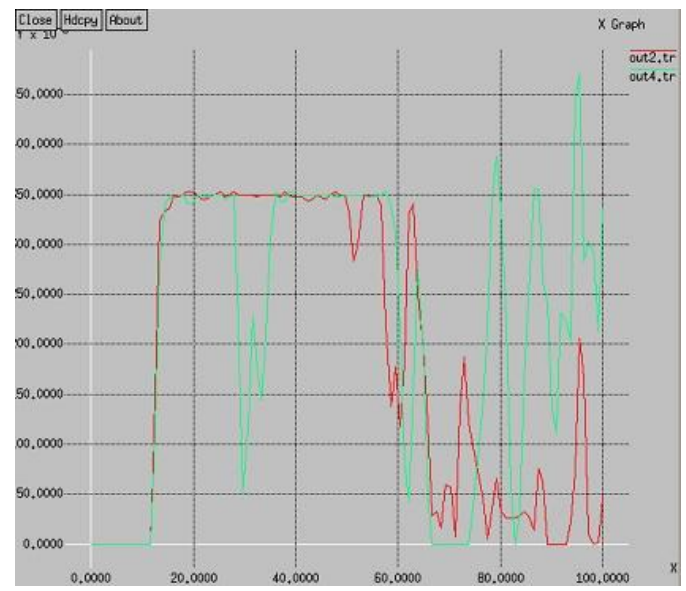

Fig. 3: Throughput

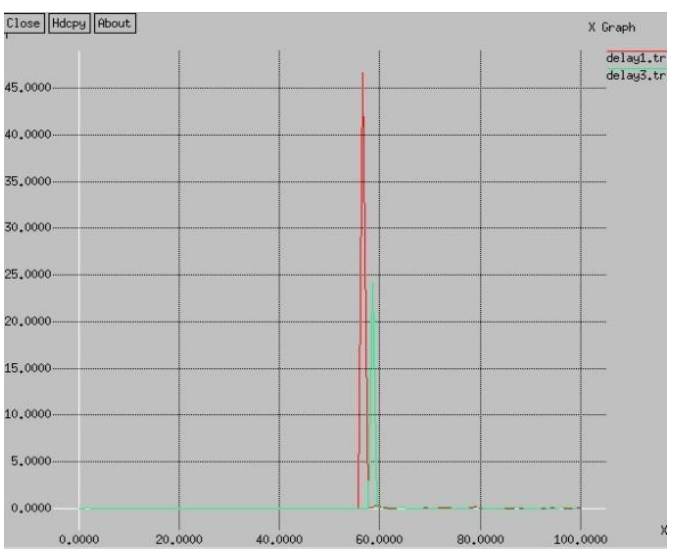

Fig. 4: Delay 


\section{ACKNOWLEDGEMENT}

I express my gratitude to my thesis counsellor Mr. Mohinder Kumar of the Department of CSE at Lovely Professional University for his continuous guidance in my study and research, for his encouragement, endurance, inspiration, keenness, and immense knowledge. His guidance helped me a lot during the period of research and preparation of this report. His good self is always ready to help me whenever I had any doubt about my research on the subject. He time and again allowed this research to be my own work, but guided me in the right way whenever he considered I required it. I must also convey my very deep gratefulness to my parents for providing me with constant support and encouragement during my whole years of study and throughout the course of researching and preparing this report. This accomplishment would not have been achievable without their support and guidance.

\section{REFERENCES}

[1] Jinhua Guo and Nathan Balon University of Michigan Dearborn "Vehicular Ad Hoc Networks and Dedicated Short-Range Communication", pp.8-13.

[2] Yang Zhang Department of Computer Science \& Engineering The Pennsylvania State University, Jing Zhao Department of Computer Science \& Engineering The Pennsylvania State University, Guohong Cao Department of Computer Science \& Engineering The Pennsylvania State University "On Scheduling VehicleRoadside Data Access”, pp.1-5.

[3] Sarita Negi, Smita Singh, Akshay Kumar Department of Computer Science and Enggineering, Akshay Kumar GBPEC, Pauri Garhwal Department of Electrical Engineering GBPUA\&T "Data scheduling in VANET:A survey" Pantnagar, India, International Journal of Computer Science Trends and Technology (IJCST) Volume 4 Issue 2, Mar - Apr 2016, pp. 25-30.

[4] Yong Hao, Jin Tang, Member, IEEE, and Yu Cheng, Senior Member, IEEE, "Secure Cooperative Data Downloading in Vehicular Ad Hoc Networks", IEEE Journal on Selected Areas in Communications/Supplement, Vol. 31, No. 9, September 2013, pp.523-525

[5] Xu Liya, Deng Anyuan, Gao Guanyong, School of Information Science and Technology, Jiujiang University, Yang Wenzhong, School of Information Science and Engineering, Xinjinag University and Cheng Yong, School of Computer and Software, Nanjing University of Information Science and Technology "An Efficient Routing in Urban Vehicular Ad Hoc Networks" International Journal of Smart Home Vol.10, No. 8 (2016) pp.190-195

[6] Tan Yan, Wensheng Zhang, Member, IEEE, and Guiling Wang, "DOVE: Data Dissemination to a Desired Number of Receivers in VANET", IEEE Transactions On Vehicular Technology, Vol. 63, No. 4, May2014 pp.1903-1909.

[7] Neeraj Kumar and Jong-Hyouk Lee, Senior Member, IEEE, "Peer-to-Peer Cooperative Caching for Data Dissemination in Urban Vehicular Communications".
[8] H. Su and X. Zhang, "Clustering-based multichannel MAC protocols for QoS provisionings over vehicular ad hoc networks," IEEE Trans. Veh. Technol., vol. 56, no. 6, pp. 3309-3323, Nov. 2007.

[9] Khaled Ibrahim B.S. June 1999, Alexandria University, Egypt M.S. January 2004, Alexandria University, Egypt "Data aggregation and dissemination in vehicular ad-hoc networks" pp.1-5.

[10] Brij Bihari Dubey, Naveen Chauhan and Prashant Kumar Department of Computer Science and Engineering National Institute of Technology Hamirpur (H.P.) INDIA, "A Survey on Data Dissemination Techniques used in VANETs", International Journal of Computer Applications (0975 - 8887) Volume 10- No.7, November 2010 pp.5-9

[11] Xia Shen, Student Member, IEEE, Xiang Cheng, Senior Member, IEEE, Liuqing Yang, Senior Member, IEEE, Rongqing Zhang, Student Member, IEEE, and Bingli Jiao, Member, IEEE "Data Dissemination in VANETs: A Scheduling Approach" IEEE Transactions on Intelligent Transportation Systems, Vol. 15, NO. 5, October 2014, pp.2213-2215.

[12] Khaleel Mershad and Hassan Artail "A Framework for Secure and Efficient Data Acquisition in Vehicular Ad Hoc Networks", IEEE Transactions On Vehicular Technology, Vol. 62, No. 2, February2013,pp.536-540.

[13] V. Namboordiri and L. Gao, "Prediction-based routing for vehicular ad hoc networks," IEEE Trans. Veh. Technol., vol. 56, no. 4, pp. 2332-2345, Jul. 2007.

[14] L. Wischhof, A. Ebner, H. Rohling, M. Lott, and R. Halfmann, "Adaptive broadcast for travel and traffic information distribution based on inter vehicle communication," in Proc. IEEE IV, Jun. 2003, pp. 6-11.

[15] G. Korkmaz, E. Ekici, and F. Ozguner, "Urban multi-hop broadcast protocols for inter-vehicle communication systems," in Proc. ACM VANET, Oct. 2004, pp. 76-85.

[16] K. Shafiee and V. C. M. Leung, "A reliable robust fully ad hoc data dissemination mechanism for vehicular network," Int. J. Adv. Sci. Technol., vol. 2, pp. 53-62, Jan. 2009.

[17] V. Naumov and T. R. Gross, "Connectivity-aware routing (CAR) in vehicular ad hoc networks," in Proc. IEEE INFOCOM, May 2007, pp. 1919-1927.

[18] N.Wisitpongphan, F. Bai, P. Mudalige, V. Sadekar, and O. Tonguz, "Routing in sparse vehicular ad hoc wireless networks," IEEE J. Sel. Areas Commun., vol. 25, no. 8, pp. 1538-1556, Oct. 2007.

[19] V. Namboordiri and L. Gao, "Prediction-based routing for vehicular ad hoc networks," IEEE Trans. Veh. Technol., vol. 56, no. 4, pp. 2332-2345, Jul. 2007.

[20] R. He, H. Rutagemwa, and X. Shen, "Differentiated reliable routing in hybrid vehicular ad-hoc networks," in Proc. IEEE ICC, May 2008, pp. 2353-2358. 